\title{
Frontières
}

\section{Rendre son dû à la Peur}

\section{Pierre Migneault}

Volume 12, numéro 2, printemps 2000

Peur bleue...

URI : https://id.erudit.org/iderudit/1074393ar

DOI : https://doi.org/10.7202/1074393ar

Aller au sommaire du numéro

Éditeur(s)

Université du Québec à Montréal

ISSN

1180-3479 (imprimé)

1916-0976 (numérique)

Découvrir la revue

\section{Citer cet article}

Migneault, P. (2000). Rendre son dû à la Peur. Frontières, 12(2), 14-19.

https://doi.org/10.7202/1074393ar

\section{Résumé de l'article}

Un texte d'évocation et de sensibilisation à l'importance de la Peur dans l'économie psychique individuelle et collective. On a généralement peur de la Peur, pourtant un guide et allié fort bénéfique. La Peur, comme grille de lecture et compréhension des institutions et systèmes religieux, politiques, scientifiques, psychologiques... avec risque de dérives, comme le consortium de la peur. En arriver à mieux vivre avec les aspects mobilisateurs et positifs de la peur.
Ce document est protégé par la loi sur le droit d'auteur. L'utilisation des services d’Érudit (y compris la reproduction) est assujettie à sa politique d'utilisation que vous pouvez consulter en ligne.

https://apropos.erudit.org/fr/usagers/politique-dutilisation/ 


\section{Rendre son dû à la Peur}

“IL FAUT RÉAPPRENDRE À AVOIR PEUR»

\section{Résumé}

Un texte d'évocation et de sensibilisation à l'importance de la Peur dans l'économie psychique individuelle et collective. On a généralement peur de la Peur, pourtant un guide et allié fort bénéfique. La Peur, comme grille de lecture et compréhension des institutions et systèmes religieux, politiques, scientifiques, psychologiques... avec risque de dérives, comme le consortium de la peur. En arriver à mieux vivre avec les aspects mobilisateurs et positifs de la peur.

Mots clés: peur - angoisse - désarroi

\begin{abstract}
This text serves as a recapitulation of and sensitization to the scope of Fear in the individual and collective psychic economy. We generally fear Fear, nonetheless a quite beneficial guide and ally. Fear acts as a reading and comprehension gauge for religious, political, scientific and psychological institutions and systems.... with risk of downfall, such as fear consortium. How to manage to live better with fear's stirring and positive aspects.
\end{abstract}

Key words: fear - distress - dismay

- C.G. JUNG ${ }^{1}$

\author{
Pierre Migneault, \\ psychiatre à l'Hôpital Douglas.
}

\section{INTRODUCTION, AVEUX PRÉALABLES ET LEXIQUE}

Décaper, restaurer la Peur (et ses avatars) dans toute sa belle nudité et crudité, dans toutes ses nobles et redoutables fonctions. Voilà mon objectif.

Quels sont le rôle et la place de la Peur, des peurs, dans la nature et dans l'économie psychique individuelle et collective, à partir de ma propre expérience, personnelle et clinique?

Voilà ma question-gigogne, ma «nécessité intérieure», pour reprendre la belle expression de Kandinsky², le sujet qui me porte plus que je le porte.

L'énigme de la Peur a probablement d'ailleurs largement présidé à mon choix de devenir psychiatre, une façon subtile et socialement acceptable de se rapprocher de ses peurs, de sa souffrance, de son désarroi, de ses zones d'ombre et de lumière, de les amadouer, de les explorer, tout en s'occupant de celles des autres. Jean-Yves Roy a très bien esquissé ce thème délicat ${ }^{3}$ et Boris Vian l'a vraiment dépecé, avec son personnage de Jacquemort dans l'Arrache-coeur ${ }^{4}$

Un métier dangereux. On risque même, comme psy, de succomber aux charmes de la Peur, et de son compagnon d'infortune, le Désarroi, ce qui est un peu mon cas 5 .

Le lexique, le vocabulaire généré par la Peur est d'une abondante et riche diversité, qu'il s'agisse du langage populaire, poétique ou savant. Tout cela confirme la force évocatrice de cette onde de choc qu'est la Peur et de sa forme exacerbée, l'Angoisse. Il suffit de consulter, au vocable peur, un dictionnaire analogique, pour constater les dizaines de nuances, de tonalités différentes. On y définit la peur comme un sentiment d'inquiétude éprouvé en présence ou à la pensée d'un danger. On partira de là.

La Peur me paraît le terme générique le plus ancien, le plus évocateur et partant, le plus approprié. Même les enfants la ressentent, la recherchent, la comprennent. "Maman, j'ai peur».

La Peur et ses dérivés, dont l'Angoisse ou mieux encore l' «angoissement» pour reprendre le terme plus générique proposé par Diel ${ }^{6}$, prennent des significations et appellations fort différentes selon leur champ d'application ou d'observation: anthropologique, littéraire, esthétique, religieux, philosophique, psychologique, psychanalytique, sociopolitique, biologique ou médico-psychiatrique.

L'Angoisse, dans le champ médicopsychiatrique qui est le mien, a une composante, une résonance physique plus explicite. Elle est une peur imaginative avec impossibilité apparente de décharge satisfaisante.

L'Angoisse est un dérivé beaucoup plus complexe que la Peur qui, elle, est plus immédiate, une sorte de réactivité automatique déclenchée par une menace réelle ou imaginaire. Une voie réflexe plus courte $^{7}$, comme l'intuition d'ailleurs ${ }^{8}$.

L'Angoisse est l'indice d'un dérèglement de l'harmonie d'ensemble des fonctions intra-psychiques, dérèglement qui se reflète sur le plan endocrinien et qui produit aussi des troubles somatiques ${ }^{9}$.

Quant au Désarroi, du vieux français "arroyer», il évoque davantage, comme une sorte de voie finale commune, la difficulté de comprendre, d'arrimer les choses ensemble pour leur donner un semblant de sens ou de cohérence. Il implique diverses fonctions intellectuelles supérieures. Il va à l'essentiel par des chemins fort détournés.

Le Désarroi, un vocable de genre masculin. Curieux et intéressant. C'est à 
se demander si les genres masculin et féminin, en langue, n'auraient pas entre autres quelque chose à voir avec cette intuition fondamentale, viscérale, à savoir qu'il y a une polarité de registre chez l'humain: un registre affectif, instinctuel, émotif et un registre plus rationnel, plus imaginatif, le lieu des pourquoi et des ébauches de réponses, le lieu d'élaboration des grands schémas explicatifs et des grands récits. "Père, Père, pourquoi m'as-tu abandonné?»

La Peur, l'Angoisse ou l'Angoissement, l'ensemble des formes d'angoisse, et le Désarroi, une sorte de triptyque, seront les trois éléments de base ou balises de mon travail.

La Peur, avec une majuscule, désignera le phénomène en général, avec son arrière-fond tout en verticalité; la peur, avec minuscule, indiquera une peur plus spécifique, mieux identifiée, plus dans l'ordre horizontal, visible, concret des choses. La peur de la maladie, par exemple.

Il en ira de même pour tous les autres dérivés ou nuances de la Peur, en majuscule ou minuscule. Le registre minusculaire, c'est là où les choses se déroulent, se voient, se palpent, se mesurent. Le registre majusculaire, c'est là où les choses se passent vraiment, dans le moins visible, voire dans le mystère le plus total. Être père, mère, ça se voit mieux: la Maternité, la Paternité, où ça se loge?

Ce travail comportera quatre parties ou étapes, comme un rituel de sensibilisation, avec un thème récurrent presque grégorien: l'éloge prudent de la Peur et de ses acolytes.

J'utiliserai des mots et surtout des images qui camouflent moins et rendent mieux la réalité. Cette conjonction de mots et d'images, comme dirait un compagnon d'Ulysse, permet d'accoster avec plus de sécurité dans l'antre fascinant et redoutable de la Peur. À chacun ses moyens de séduction.

\section{RECONNAÎTRE L'OEUVRE DE PEUR EN SOI}

Il est plus facile de parler de la Peur en général, surtout celle des autres, que d'aborder sa Peur propre et surtout de l'expérimenter en soi. Pourtant il n'existe pas d'autre façon de rejoindre le courant du dynamisme transformateur de la Peur et de l'Angoisse. Il faut d'abord ramer, seul et dur, en soi, affronter et traverser ses peurs, comme un "cerceau de feu».

Le chemin le moins facile, le "moins fréquenté» souligne pertinemment Scott Peck $^{10}$, c'est celui qui part de soi, de l'expérience personnelle, et qui y retourne ultimement.

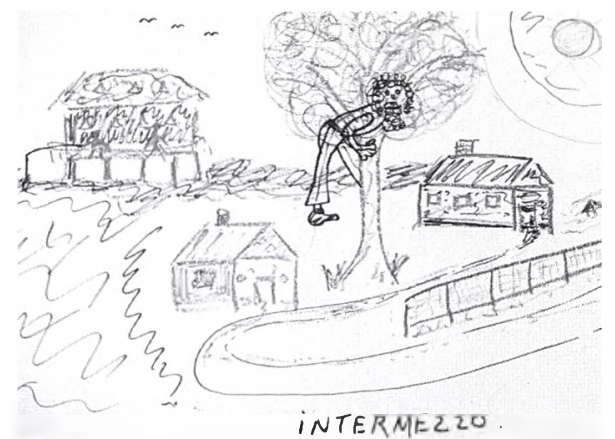

Un patient psychotique d'une trentaine d'années se décrit, agrippé à sa peur, à la suite de l'interruption d'un groupe d'expression graphique auquel il était très attaché.

C'est là, dans le repaire privé du soi que se manifeste, avec le plus d'exigence, la peur extrême, à mon avis, la peur de soi, le point de départ et la voie finale commune de toutes les peurs. En d'autres mots, ce qui effraie surtout, c'est la peur des réactions émotives que les objets ou motifs de peur, réels ou imaginés, déclenchent en chacun de nous. Peur des émotions et mouvements d'humeur ainsi suscités (violence, haine, idéation suicidaire, honte etc.).

J'ai peur surtout de ce que les peurs m'apprennent sur moi, sur ma vulnérabilité, sur mes faiblesses et lâchetés, sur mes dysharmonies intérieures, mes contradictions, mes refoulements. La Peur m'oblige à faire des remaniements constants de mes rapports biscornus au monde ambiant et surtout à moi-même.

"Rendu à tout, on retombe à soi et puis à rien", constate magnanimement Jacques Ferron ${ }^{11}$, au creux de son superbe désarroi, du fond de son arrière-cuisine, l'espace privilégié du "Moi crucifiant».

Les Mânes de Ferron me fascinent et me troublent. Ceux aussi de Dostoïevski12, surtout celui des Carnets $d u$ sous-sol, relatant la peur et la difficulté de vivre avec soi-même.

Il faut d'abord, à mon humble avis, rencontrer, reconnaître, assumer Phobos, la Peur, cette grosse pierre qui bloque l'entrée de soi, si l'on veut arriver justement à faire le tour de soi et de ses peurs, passage obligé vers l'émerveillement et la joie de vivre avec moins de lambeaux de soi laissés au vestiaire ou aux oubliettes honteuses. Tout un contrat de vie entière, sans guide assuré.

Les vertus exaltantes et les vices dégradants, apeurants, sont parties prenantes de la vie et de nous-mêmes. La Peur est une leçon vivante de complexité. Elle génère le meilleur et le pire. Elle constitue, à mon avis, la leçon de vie par excellence, l'expérience fondatrice de la condition humaine.

L'homme est "une plante dont les racines sont au Ciel», proclamait avec lucidité Novalis. Qu'y a-t-il d'étonnant à ce que la Peur soit à la fois son lot et son atout le plus précieux?

C'est ma conviction la plus profonde et le leitmotiv de ce texte. C'est aussi, il faut bien l'avouer en fin de parcours, ma plus grande source d'étonnement et de frustration, comme psychiatre. En clinique, on rencontre en effet la plupart du temps des gens en difficulté qui veulent éviter, anesthésier au plus vite et à tout prix, leur Peur, leur Angoisse, leur Désarroi, pourtant nos meilleurs alliés, signaux d'alarme et guides, autant pour le patient que pour le thérapeute qui, hélas, tombe souvent dans le même affolement ou affairement autour de son propre Désarroi soudainement réveillé, en écho. Complexe.

\section{L'ÉNIGME DE LA PEUR}

Comment se fait-il que nous ayons tant de mal à lui faire face et à lui laisser sa place, à cette Peur-Vigie, cette PeurVie, cette Peur-Îsha ${ }^{13}$ qui assume pourtant plusieurs fonctions vitales?

La peur et l'angoisse, son arrièrefond plus voilé, sont un phénomène central de la vie et de son évolution. L'angoisse coupable révèle, selon Diel, les péchés contre nature, du côté de la banalisation, du pervertissement ou de l'exaltation imaginative. On n'aime pas que la Peur nous les rappelle?

Sous la houlette herméneutique et tendre de Paul Diel ${ }^{14}$, les récits bibliques deviennent de merveilleuses et troublantes leçons symboliques de vie, avec toutes ses tentations et pièges. On $y$ retrouve, à peine déguisée, toute la gamme psychopathologique humaine: l'angoisse, les troubles de l'humeur, les troubles de la pensée et du comportement.

L' "énigme de la peur», souligne aussi Henning Kohler ${ }^{15}$, réside surtout au niveau de la peur de la peur, une sorte d'expansion de la peur qui nous prive de tous ses pouvoirs bénéfiques de prise de conscience et de transformation, la crise panique en constituant la manifestation ou crispation la plus inhibitrice. Au lieu d'agir, de réagir de façon appropriée ou de fuir, on rentre dans une sorte de léthargie panique.

Kohler, ce pédagogue d'orientation anthroposophique oeuvre en Allemagne, dans un Institut pour enfants handicapés. Il soutient, avec beaucoup de perspicacité, que la peur est parfois le signe d'une trop grande ouverture au monde et qu'elle peut être métamorphosée en qualités précieuses de tact et de sensibilité.

La peur, suggère-t-il, est un signal individuel d'alarme et d'effroi - Freud 
l'avait déjà bien rappelé - mais aussi un questionnement de notre rapport erroné au monde. Hypothèse fort intéressante.

\section{J'AI PEUR, DONC JE VIS}

La peur, c'est un peu comme le tonus de base de tout être vivant et conscient, le tonus musculaire étant l'état de légère contraction permanente du muscle vivant.

La clinique psychiatrique vient, avec toutes ses manifestations exacerbées de la Peur, dont l'anxiété panique, corroborer cette assertion. La Peur sous-tend et accompagne la folie et les dérèglements physico-psychiques, sous toutes leurs formes.

De là la peur, l'effroi, que réfléchit, comme dans un miroir déformant, grossissant, la folie. Personne n'en est dupe. Les Monomanes de Géricault en sont une troublante illustration. On raconte qu'une large bannière affichant ce Monomane et surplombant une voie publique fort achalandée, lors d'une grande exposition à Paris, il y a quelques années, a dû être enlevée.

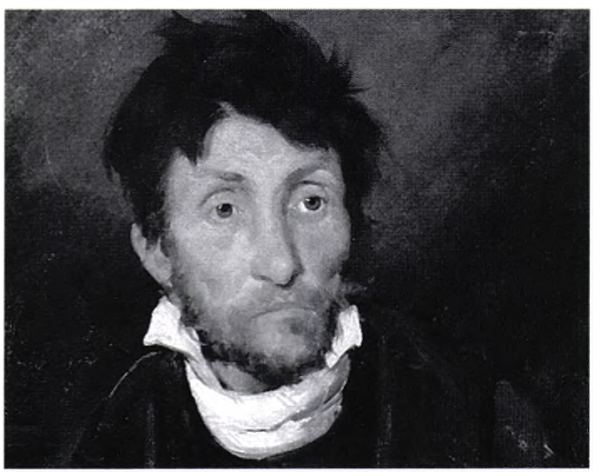

Théodore Géricault, Le Monomane, 1822

La circulation routière, au rythme échevelé, ralentissait trop... Comme si la conjonction Art et Peur était trop puissante et dangereuse.

La Peur, le dés-arroi, dans le sens du dés-arrangement ou nécessaire désordonnance du sens et dé-construction de l'ordre des choses, est le lot commun et la caractéristique ultime de toute matière vivante consciente.

La mythologie universelle, les contes et légendes tous azimuts, les récits bibliques de la Création, la panoplie des dieux, relatent, témoignent de la Peur et des peurs et essaient d'y répondre, de les calmer.

La Peur est la dernière sorcière que l'on essaie d'amadouer, lors de l'endormissement et elle continue, comme une lampe du sanctuaire, à meubler, à hanter et à ratisser nos nuits, à rétablir l'équilibre instable et nécessaire entre l'ordre et le désordre.

La Peur nocturne veille surtout, comme les singes-vigies de Bruegel, à remettre en contact, entre eux, les divers registres de notre être soigneusement dissociés, déconnectés, durant l'état de veille, image et rectitude publique obligeant.

La Peur accompagne le premier cri humain, à la naissance, comme elle lâche prise seulement avec le tout dernier souffle de vie. Elle est la première visiteuse du matin qui annonce le réveil, quand la respiration ne relève plus du pilote automatique. La première confirmation d'une survie toujours étrange et joyeuse. Il y avait la nuit, il y a le matin.

La Peur viscérale, endémique, ontologique, on la ressent constamment à fleur de peau, surtout durant l'apnée, au sein de chaque cycle respiratoire et de chaque cycle cardiaque. On la porte constamment en bandoulière, comme les personnages apeurés et joyeux de Bruegel.

La Peur, les peurs, sont finalement la meilleure amie et alliée de l'être humain. La peur fonde, tonifie le rythme humain vital de base. À partir de là, toutes les réalisations, impulsions et dérives comportementales humaines sont possibles et relativement compréhensibles.

La Peur s'avère être une "grille de lecture» incontournable pour la saisie et l'abordage des phénomènes humains les plus complexes. Il faudrait rendre un hommage respectueux à la Peur, comme les enfants savent si bien le faire, ce que les adultes, devenus raisonnables, hélas, envient ou ne savent plus très bien faire.

Réjane Charpentier, dans ses textes pour théâtre d'enfants, nous le rappelle de façon absolument fascinante et convaincante ${ }^{16}$.Un véritable récit de création où la peur trouve ou prend, comme la liberté, sa juste place et son rôle. Les enfants ne s'y trompent pas et jubilent, pendant que les adultes (parents, enseignants, etc.) s'inquiètent.

Le langage et l'imagerie populaire, quant à eux, n'en finissent plus d'inventer des termes colorés, étripants - la trouille, la chiasse, etc.- au point d'en "avoir chaud aux fesses".

La Peur déniaise, dépucelle, heurte et renforce aussi l'âme, déclenche la difficile et nécessaire perte de l'état d'innocence: elle nous oblige enfin à aborder crûment toute la réalité, entre ange et bête; on peut ensuite mieux composer avec toutes les autres émotions fortes ou dangereuses.

Les rituels d'apprentissage shamanique utilisent beaucoup les situations de peur ${ }^{7}$; il en va de même pour les divers rituels de passage chez les Amérindiens ${ }^{18}$. L'apprentissage psycho- thérapeutique, la sortie de l'exil de soi, le pire exil en fait -, et la traversée de notre ombre, passent inévitablement par l'expérience de la Peur.

La Peur révèle, à mon avis, le sens profond, voilé, du registre blasphématoire; on ne blasphème pas toujours par manque de vocabulaire mais plutôt sous l'effet grisant d'un mélange complexe de fascination et de tremblement, d'effroi sacré devant l'inconnu, devant l'insoutenable beauté et laideur énigmatique du monde, de la vie.

"Ça n'a pas d'ostie.*** de bon sens comme c'est beau, que c'est bon et difficile, la vie, foi de Jésus-Christ!», s'exclamait, en riant de larmes, un patient schizo-affectif fréquentant un groupe d'expression artistique, très fier de dire, en sacrant toujours, qu'à trente-cinq ans, il avait dépassé l'âge du "Christ» et que le Christ le jalousait, Lui, un Dieu qui avait "lâché prise" à trente-trois ans!

À travers ses dessins d'enfant, il se décrivait comme un Petit Prince Martien auquel des dieux désemparés devant leur Création avaient greffé un cœur humain, à titre d'ultime essai d'ailleurs infructueux, selon lui. Il se donnait comme exemple de cette impossible opération, son "Incarnation" ratée. Job blasphèmant.

On arrive, plus ou moins à corps défendant et sur trois pattes, comme Oedipe, à déboucher ou à se résigner à dire: Amen, Alléluia. En arriver à intégrer le registre de la colère infinie, du désarroi et du ressentiment global, à travers la cacophonie du blasphème, en arriver à l'Alléluia-Tabarnak, comme Marcel Saucier ${ }^{19}$, avec toutes ses variantes et bémols, c'est beaucoup plus difficile, à mon avis.

La Peur, c'est surtout le signe d'un manque de foi en son corps, en soimême, en sa capacité de se relier au monde ambiant et d'y trouver sa place.

\section{AU COMMENCEMENT ÉTAIT LA PEUR... QUELQUES LEÇONS D'HISTOIRE}

L'histoire et l'inventaire des dérives et aberrations imaginatives ${ }^{20}$ légitimement commises, au nom et sous le prétexte de réponses à la peur, sont riches d'enseignement et souvent accablants. On parlera de la Bible, de l'apport de la famille Ellenberger et de l'œuvre de Pfister, avec un clin d'œil à Éric Berne.

«J'AI PEUR, CAR JE SUIS NU

ET JE ME CACHE» (Adam)

La Peur ontologique se révèle bien dans l'histoire fondatrice du Paradis terrestre. On y retrouve, sous déguise- 
ment, le complexe fondamental de l'homme-Dieu et du Dieu fait homme. On n'en sort pas.

Un clone humain, Adam, encore inaccompli, inachevé, impatient, idiot, fournit, sous le prétexte de la pomme interdite, à un Dieu lui-même dépité devant sa Création inachevée, inachevable, le plus bel alibi et l'occasion de passer le blâme à sa propre Créature.

Le passage à relais du blâme de la Création inachevée, la Création inaccomplie de soi en constituant l'exemple type, se répète inlassablement selon le modèle de cette scène fondatrice.

L'exemple de l'évitement du blâme et de la responsabilité vient de haut et de loin. Créatures humaines et divines ont un trait en commun, la Peur. Les récits de la Création ne s'y trompent pas.

Le doigt accusateur du Dieu offusqué pointant l'Adam, le terrien, refilant ce blâme à Ève, son Ishah, l'autre partie de lui-même. Ėve renvoie le blâme au serpent, diviseur-séparateur du Soi, qui le retourne à sa source, le Très-Haut. Suivons la courbe et direction du doigt accusateur: seule la Peur peut susciter et nous faire gober un tel récit à la fois merveilleux et piégé.

«Délirer de peur», écrivait Georges Bataille, peu de temps avant de mourir. La Peur «fonde» les grands récits, les grandes références et institutions fondatrices, pour reprendre l'expression heureuse de Pierre Legendre qui a très bien dépisté l'oeuvre de Peur et la crise des référents, au Québec, à travers le crime mythique du Caporal Lortie ${ }^{21}$.

En bref, le prototype du plus fondamental mécanisme d'évitement de la Peur et d'évitement de Soi, par personnes interposées, impliquant autant les Dieux que les humains, le règne végétal et animal, est là, gravé dans le bronze, comme il se doit sur les portes d'une cathédrale, lieu privilégié des questions fondamentales sans réponses.

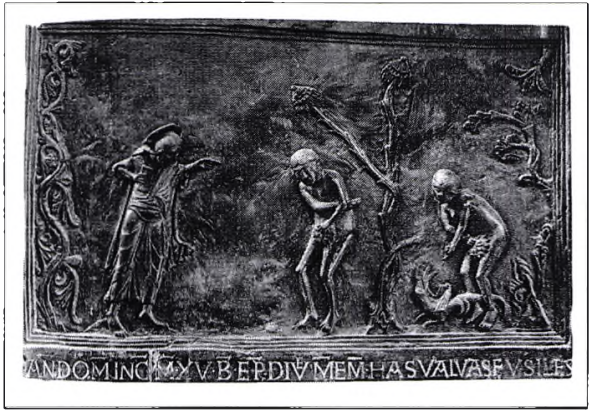

Adam and Eve Reproached by the Lord, 1015, portes de bronze, St.-Michael's Church, Cathedral Hildesheim, Allemagne

La lecture métaphorique des Récits de la Création, comme celle qu'en fait Jean Rousseau 22 redonne accès, para- doxalement, à la richesse infinie de cette plus belle histoire du monde dont justement, une lecture généralement trop timorée ou apeurée, trop métonymique, nous avait souvent écartés, en la dénaturant de son pouvoir évocateur.

Dommage qu'on se soit souvent servi de ces récits et mythes de Création pour faire peur et justifier une immense entreprise contra-phobique, l'organisation religieuse, aux multiples fonctions aussi nobles que pernicieuses. La vigilance est de rigueur.

Que les aînés se souviennent de cette savante opération peur-en-crescendo des retraites fermées québécoises, avec décompression et pardon final, après le rituel de confession, bien sûr.

Les despotes, les tyrans, les tortionnaires, les gourous de tout acabit, incluant les psys et leur Inconscientépouvantail et certaines de leurs terribles simplifications, bref tous les êtres de pouvoirs utilisent souvent la même denrée de base, la Peur. Faire peur pour régner.

Une observation prolongée de cette scène primitive - la vraie à mon avis et non l'autre si souvent citée, la diversion Oedipienne -, amène son spectateur à adopter exactement le sourire énigmatique de La Joconde. Il est peut-être là le secret de Léonard?

Cette oeuvre d'art témoigne aussi de la puissance de l'expression artistique, qui, rehaussée d'humour sacré, est peutêtre la voie réparatrice finale commune de la condition humaine dépitée, apeurée, une fois cette Peur assumée et remise majestueusement à sa juste place, à la Bruegel, comme dans la Chute d'Icare?

La foi en soi, l'art de penser, de vivre, de s'exprimer et la capacité de rire de soi et de la vie constituent peut-être la trilogie réparatrice par excellence de la Peur?

\section{LA FAMILLE ELLENBERGER ET LA PEUR}

Un bel exemple d'observation des répercussions individuelles et sociales de la Peur ancestrale vient de la contribution de la famille Ellenberger.

Les nombreux travaux d'Henri F. Ellenberger (1905-1993) sont bien connus $^{23}$. Il relate très bien l'évolution et les liens entre les divers systèmes explicatifs et approches psychothérapeutiques depuis l'animisme africain à la psychanalyse et toutes les autres réponses psy au désarroi d'époque.

Il évite les jugements de valeur, les conflits d'école et s'attache surtout à contextualiser et à imbriquer les uns dans les autres ces discours et approches psy plus complémentaires que conflictuelles et qui, au fond, sont autant de réponses à la Peur antique et toujours aussi persistante, le véritable fil conducteur, selon lui, de l'évolution des systèmes de pensée.

Le grand-père, David Frédéric Ellenberger, et le père, Victor, étaient missionnaires protestants en Afrique centrale et purent ainsi très bien observer, entre 1860 et 1920 , les populations locales.

Victor Ellenberger ${ }^{24}$ rédigea en 1958 un livre très intéressant et troublant, une sorte de bilan de sa vie: Afrique, avec cette peur venue $d u$ fond des âges: sorcellerie, initiation, exorcisme. "Au commencement était la peur...", écrit-il tout au début du livre.

La peur suinte de partout, des phénomènes climatiques et naturels, de la famine, de la violence tous azimuts, des guerres, de l'emprise des dirigeants, des divers rituels, des féticheurs, des devins, des sorciers..

L'évangélisation missionnaire, le progrès de la civilisation, consiste, selon le missionnaire européen, à sortir la population africaine de l'emprise de la peur. "Au carrefour des pistes obscures de la jungle païenne»[...], "une institution de clémence et d'espoir» (l'Église protestante). Encore une fois, on croyait honnêtement avoir trouvé la réponse.

En guise ultime de preuve du succès de cette entreprise missionnaire, le shaman dominant se convertit... avouant finalement avoir "trompé» la population avec «son chapelet d'osselets divinatoires». Victor Ellenberger était rassuré.

On y voit, à l'oeuvre en Afrique, tous les liens de compétition et de complicité entre les tenants des divers pouvoirs d'époque (politique, religieux, le savoir du guérisseur etc.). La Peur et tous ses avatars y jouent le rôle prédominant: Victor Ellenberger observe et déplore qu'on suscite la peur, qu'on l'entretienne, l'explique, l'exploite, et qu'on propose ensuite des moyens plus ou moins irrationnels de la soulager, de l'extirper.

Chaque époque, chaque groupe ou collectivité humaine, selon son niveau de développement, ne donne-t-il pas naissance à une sorte d'entreprise ou consortium de la Peur dont le rôle est d'élaborer des réponses aux peurs courantes, des right-wrong answers?

Le progrès de la civilisation, les développements scientifiques et technologiques, la répartition des biens et richesses, les divers modes de gouvernement, fournissent des outils et des réponses différentes à la Peur mais l'indice de détresse, pour utiliser une nouvelle échelle moderniste, est loin de diminuer, même dans notre coin de planète à révo- 
lution tranquille, où nous n'avons pourtant connu véritablement «ni la faim, ni la guerre», disait Claude Jutras.

Les plaques tectoniques de la Peur continuent, sous des formes différentes, à faire leur oeuvre souterraine. En habits et sous formes et appellations différentes, ne pourrait-on pas très bien retracer des affinités et ressemblances avec les acteurs actuels de cet immense consortium des réponses à la Peur contemporaine?

Le risque permanent de la disqualification et de l'exploitation de l'humain par l'humain, au nom de la Peur, se retrouve à travers toute l'histoire humaine.

\section{CHRISTIANITY AND FEAR:}

OSKAR PFISTER ${ }^{25}$

Je terminerai cette série d'exemples historiques avec cette mention d'une oeuvre fort pertinente pour la prospection des «oeuvres» de Peur.

Ce qu'il y a de fascinant et de troublant avec Oskar Pfister (1873-1956), qui a bien connu Jung, qui a maintenu une longue correspondance et amitié avec Freud (1909-39) ${ }^{26}$ et qui fut aussi l'analyste d'Henri F. Ellenberger, c'est qu'il est presque contemporain; qu'il a baigné dans les mêmes apports, illusions et désillusions des grands systèmes de pensée philosophiques, religieux, politiques, scientifiques, etc. modernes. Il est bien au fait des apports et critiques de l'Irrationalisme contemporain: Schopenhauer, Nietzsche, Freud, Adler, Jung, Sartre ${ }^{27}$.

Empruntant avec une ferveur étonnante, pour ne pas dire suspecte, les notions psychanalytiques freudiennes ${ }^{28}$, ce pasteur protestant, devenu analyste d'âme, stigmatise la dégradation et le pervertissement du message d'amour de Jésus-Christ depuis deux mille ans, par les diverses organisations judéo-chrétiennes.

La Peur, encore elle, à preuve l'Inquisition, a remplacé selon lui l'Amour et il faut renverser le mouvement et revenir au message initial. La Peur, normale et névrotique, constitue sa grille de lecture et de compréhension de l'histoire des grandes religions, avant et après Jésus-Christ.

Le dénonciateur-réformateur, en l'occurrence Oskar Pfister, se substitue à ou remplace l'erreur dénoncée plus qu'il ne la dissipe. Ce faisant, il nous livre, par la bande, une vue impressionnante, grandeurs et misères, de l'histoire des religions depuis l'avènement du Christianisme, notre germoir commun.

Ces considérations de Pfister me font enfin souvenir des remarques judicieuses d'Éric Berne ${ }^{29}$ autour de la «liberté» si souvent proclamée et revendiquée mais pas vraiment désirée, parce qu'elle fait probablement trop peur. De là, le besoin fortement ressenti d'immenses entreprises religieuses, politiques, scientifiques, économiques, etc. - de régulation de cette peur endémique de la liberté (de penser, d'agir, liberté sexuelle, etc.), concluait-il comme Pfister.

Selon Berne, on choisit le partenaire, masculin ou féminin, qu'on aime le plus, celui qu'on déteste le moins, celui qu'on sait, plus ou moins consciemment, capable d'en prendre le plus et surtout celui qui ne nous laissera jamais seul avec nous-mêmes, la peur extrême, selon Berne. L'amour et la peur.

\section{LE BEAU RISQUE DE VIVRE, ENTRE PEUR ET ÉMERVEILLEMENT: QUELQUES TROUVAILLES ET PISTES}

Je ne ferai qu'évoquer, en dernière étape, quelques trouvailles et pistes qui personnellement m'auront été très utiles, pour ne pas dire plus, autant sur le plan personnel qu'au niveau de mon travail clinique, pour aborder les manifestations de la Peur.

L'approche pluraliste de Leston $\mathrm{L}$. Havens ${ }^{30}$, psychanalyste, met l'accent sur les complémentarités plus que les conflits ou antinomies entre les divers niveaux de sensibilité menant à des écoles de pensée différentes. Quelle que soit la problématique concernée, en l'occurrence la Peur, on tente d'abord, selon Havens, de la nommer, de la classifier, ensuite de l'expliquer, de la comprendre, d'en décrire les fondements bioneurologiques. On prend ensuite du recul, une sorte de mise entre parenthèses philosophico-phénoménologique, avec la condition humaine et la finitude comme toile de fond. La Peur et ses manifestations variées deviennent alors une condition nécessaire mais insuffisante de la vie. Il ne faut jamais laisser la Peur seule avec elle-même, isolée sur ellemême.

Havens essaie de comprendre toutes les problématiques psychiques, incluant la Peur, en les situant dans un contexte relationnel humain et sociétal; il les replace dans un contexte plus global, humain, physique, cosmique. Havens débouche finalement sur une position esthético-spiritualiste de non-savoir (not knowing) et d'acceptation plus ou moins joyeuse du mystère de la chose étudiée et de la vie en général.

On pourrait très bien faire un parallèle entre ces diverses approches et styles cognitifs et les divers mouvements ou perspectives artistiques (le réalisme, le narratif, l'impressionnisme, l'expressionnisme, le conceptuel, le déconstruc- tivisme, etc). Ce n'est ni le lieu ni le temps d'élaborer davantage.

L' «approche systémale» de Pierre Marchais ${ }^{31}$ vise encore plus large en prétendant qu'il s'agit, rien de moins, de "pénétrer l'univers qui est en nous, en communication permanente avec celui qui nous entoure et dont les dysrégulations d'ensemble constituent ce que nous dénommons le trouble mental».

Appliquée au traitement, dans le sens large, de la Peur, l'approche de Marchais rappelle que la Peur et l'Angoisse sont les signaux d'alarme privilégiés de cette dysrégulation spatio-temporelle individuelle et cosmique. Un signal d'alarme pour la renégociation des rapports, en soi, entre le domaine des instincts, le domaine émotivo-affectif et intellectuel. Une renégociation similaire avec le monde ambiant. L'illustration du livre et de l'approche de Marchais, à partir d'une oeuvre charnière et intégratrice de Kandinsky, ne saurait mieux faire la «monstration" 32 de l'art de penser, de vivre, d'agir, en unification à la fois interne et au diapason avec le rythme de l'univers global.

Cette remise en contexte global de la Peur me paraît le moyen de résolution le plus efficace et pertinent, la voie artistique de la connaissance et de l'intégration de soij3 en étant l'illustration et la preuve tangible.

\section{UN REGARD ARTISTIQUE ET NAÏF}

On garde toujours, pour la fin, ses armes et secrets les plus précieux et on les révèle en s'esquivant sur la pointe des pieds.

Je perds et retrouve littéralement mes moyens et mes sens, en matière de peurs ou autres difficultés de la vie, en contemplant diverses expressions, bricolages ou productions artistiques spontanées, dont l'art naïf et sa sagesse populaire ${ }^{34}$.

"On a les yeux trop grand'ouverts pour les garder fermés», clamait Arthur Villeneuve, le peintre-barbier dit naïf. Peut-on mieux "couvrir» les divers niveaux de réalité, incluant l'espace du "sus-conscient», comme il le dénommait si judicieusement, "en continuance» avec le règne végétal, animal? La Peur s'y dissout magiquement.

Remettre la vie, et la Peur, incluant les Dieux dépités, courroucés, à leur juste place dans le vaste ordre et désordre des choses. Crier sa Peur en pleine nature et laisser les oiseaux d'Odin ou ceux du Paradis porter le message à leur Maître. Ce peintre-barbier de Chicoutimi le fait merveilleusement.

Je ne connais pas de meilleur moyen que la voie artistique non seulement 
pour arriver à survivre à ses peurs mais pour réussir à en faire le matériau jouissif par excellence.

Il faudrait, en antidote au désarroi de Job, se rabattre sur la truculence et l'irrévérence sacrée du patenteux et chefd'oeuvreux Alphonse Grenier faisant un pied-de-nez à la Mort et aux Dieux.

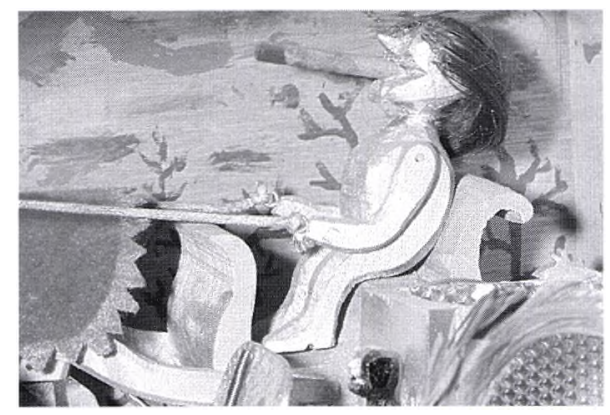

La charrette de la mort

Il faudrait lever aussi son chapeau devant le courage effronté de Marcel Saucier ${ }^{35}$. Mais on se quittera plutôt sur cette vision sereine, fière, de Marie Gélinas, grand-maman de 92 ans qui continue, dit-elle joyeusement, depuis le décès de "son homme», en 1974, à raconter et à "enjoliver» la vie, sa vie, et à remettre la Peur, et même les dieux de la Création, à leur juste place. Être redevable de son talent, être responsable de toutes les parties de soi et de la Création. Promener enfin un regard heureux sur la vie, sur soi.

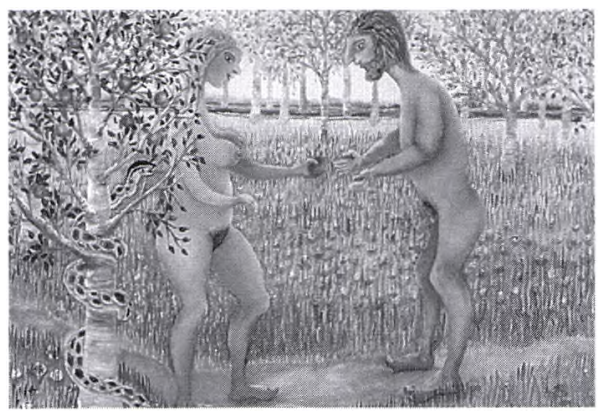

Adam et Ėve au Paradis Terrestre

«Je m'arrange avec Dieu», confiaitelle joyeusement à Josée Blanchette qui lui demandait si elle "pratiquait"sa religion ${ }^{36}$. J'ai rarement rencontré autant de santé dans les rapports à la vie, à soi, à la Peur et aux Dieux.

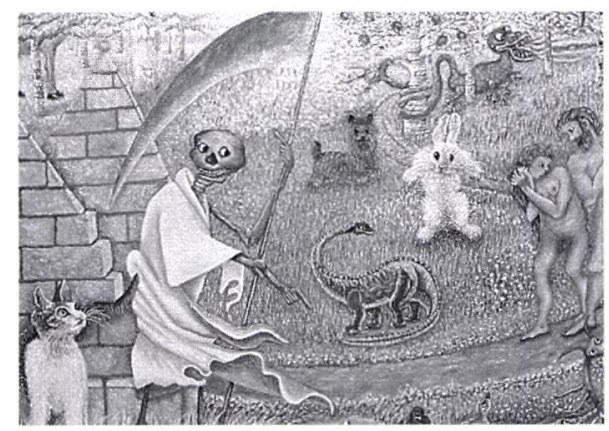

Adam et Ėve chassés du Paradis Terrestre
J'ai assez abusé de la Peur et de vous tous. Je me souhaite et vous souhaite la grâce de vivre en cocus de l'Absolu et en peureux heureux. Amen, Alléluia, Tabarnak!

\section{Notes}

1 C.G. JUNG, Word and Image: Aniela Jaffé, Bollingen Series, Princeton University Press, 1979, p. 181-187.

2 Wassily KANDINSKY, Du Spirituel dans l'art, et dans la peinture en particulier, Paris, Denoël, Folio/Essais, 1989, 215 pages, p. 2

3 Jean-Yves ROY, Être psychiatre: tentations et pièges, Éditions l'Étincelle, 1977, 136 pages.

4 Boris VIAN, L'Arrache-coeur, Paris, JeanJacques Pauvert, 1962, 256 pages.

5 Jean-Yves ROY, ibid. note 3.

6 Paul DIEL, La peur et l'angoisse: phénomène central de la vie et de son évolution, Paris, Payot, 1956, 276 pages.

7 Voir l'article de Wilfrid Noël RABY dans le présent numéro

8 Henri BERGSON, La pensée et le mouvant, Paris, PUF, 1955, c1934, 291 pages.

9 Voir DIEL (ibid. note 7) reprenant entre autres l'oeuvre de Freud.

10 Scott PECK, Le chemin le moins fréquenté: apprendre à vivre avec la vie, Paris, Robert Laffont, 1987, 378 pages.

11 Jacques FERRON, Du fond de mon arrière-cuisine, Montréal, Éditions du Jour. 1973, 291 pages.

12 Feydor DOSTOÏEVSKI, Les carnets $d u$ sous-sol, Arles, Actes Sud, 1992, 182 pages, p. 5.

13 Voir Le livre de la Création: Îsha ou Ève, la première femme.

14 Paul DIEL, Le symbolisme dans la Bible: sa signification psychologique, Paris Petite bibliothèque Payot, 1975, 238 pages.

15 Henning KOHLER, L'énigme de la peur, Montesson, Éditions Novalis, 1996, 234 pages.

16 Réjane CHARPENTIER, Coeur à Coeur (1986) et Un autre Monde (1990) pour le Théâtre de l'Oeil (compagnie montréalaise de théâtre pour enfants).

17 Henri F. ElLENBERGER, À la découverte de l'inconscient, Lyon, S.I.M.E.P., Lyon, 1976; Les mouvements de libération mythique, Montréal, Quinze, coll. Critère, 1978, 342 pages.

Victor ELLENBERGER, Afrique avec cette peur venue du fond des âges: sorcellerie, initiation, exorcisme, Paris, AmiotDumont, 1958, 350 pages.

18 Achiel PEELMAN, Le Christ est amérindien, Outremont, Novalis, 1992, 346 pages.

19 Marcel SAUCIER, Graffiti d'un calepin rose ou Les réflexions d'un miroir méchant ou Comment à vivre sans visage, on devient Dieu, poète-photographe, Malartic (Québec) inédit, 30 pages.

20 Paul DIEL, ibid. note 16.

21 Pierre LEGENDRE, Le crime du Caporal Lortie: traité sur le père, Paris, Fayard, 1989, 186 pages

22 Jean ROUSSEAU, Les Récits de la Création dans la Bible, série d'émissions radiophoniques diffusées sur Radio VilleMarie, en janvier-février 1999, 98 pages non encore publiées mais disponibles à cette station de radio montréalaise.

23 Henri F. ELLENBERGER, ibid. note 19.

24 Victor ELLENBERGER, ibid. note 19.

25 Oskar PFISTER, Christianity and Fear: A Study in the Psychology and Hygiene of Religion, London, George Allen \& Unwin Ltd., 1948, 575 pages.

26 Sigmund FREUD, Oskar PFISTER, Ernst L. FREUD et Heinrich MENG (dir. publ.) Correspondance avec le Pasteur Pfister: 1909-1939, préface d'Anna Freud, Paris, Gallimard, 1966, 209 pages.

Oskar PFISTER, Au vieil Évangile par un chemin nouveau: la psychanalyse au service de la cure d'âme pour ceux qui se sentent esclaves de leurs nerfs et de leur caractère, Berne, Ernest Bircher, 1920, 91 pages. (Avec dessins de patients en cure d'âme)

27 F.-L.MUELLER, L'irrationalisme contemporain: Schopenhauer, Nietzsche, Freud, Adler, Jung, Sartre, Paris, Petite Bibliothèque Payot, 1970, 152 pages.

28 Achiel PEELMAN, ibid. note 20. Oskar PFISTER, ibid. note 27. Sigmund FREUD, ibid. note 28.

29 Éric BERNE, Psychiatrie et psychanalyse à la portée de tous, Paris, Fayard,1971, 418 pages.

30 Leston L. HAVENS, Approaches to the Mind: Movement of the Psychiatric Schools from Sects toward Science, Boston, Little, Brown and Co., 1973, 361 pages.

Leston L. HAVENS, "The Risks of Knowing and Not Knowing», Journal Social Biol. Struct., no 5, 1982, p. 213-222.

31 Pierre MARCHAIS avec la participation d'Axel RANDRUP, Le nouvel esprit psychiatrique: métamorphose et développement de la psychiatrie clinique, Éditions Frison-Roche, 1996, 292 pages.

32 Ludwig WITTGENSTEIN, Tractatus logico-philosophicus, Paris, Gallimard. 1962, 364 pages.

33 Ibid. note 2.

34 Pierre MIGNEAULT, "L'art naïf et populaire: hommage à la vie et pied de nez à la mort», Frontières, vol. 9, no I, printemps été 1996, p. 40-42; "Le cancer, le non-sens, l'abject, la mort et l'art", Frontières, vol. 11, no 2, automne 98 - hiver 99, p. 64-66.

35 Marcel SAUCIER, ibid. note 21.

36 Josée BLANCHETTE, "La naïveté de Marie», Le Devoir, 12 mars 1999.

Je veux remercier particulièrement Lionel Beauchamp, un chercheur autonome s'intéressant à la vie et à l'oeuvre d'Henri $\mathrm{F}$. Ellenberger, qui m'a fait connaître les travaux de Victor Ellenberger et d'Oskar Pfister.

Jc veux aussi remercier Réjane Charpentier et André Laliberté du Théâtre de l'Oeil de leur collaboration éclairée et éclairante. 